\title{
Using of the Generalized Special Relativity (GSR) in estimating the proton (nucleon) mass to explain the mass defect
}

\author{
Mahmoud Hamid Mahmoud Hilo, ${ }^{1,2}$, M. D. Abd Allah ${ }^{3}$, Khalid Mohammed Haroon ${ }^{1}$ \\ ${ }^{1}$ Department of Physics, Faculty of Education, Al-Zaiem Al-Azhari University, Om Durman, Sudan \\ ${ }^{2}$ Department of Physics, Faculty of Science and Arts at Al-Rass, Qassim University, Al-Qassim, KSA \\ ${ }^{3}$ Department of Physics, Faculty of Science, Sudan University of Science and Technology, Khartoum, Sudan; ${ }^{*}$ Corresponding Author: \\ mhhlo@qu.edu.sa
}

Received 8 November 2010; revised 10 December 2010; accepted 15 December, 2010.

\begin{abstract}
This paper presents theoretical investigation on explanation of the mass defect estimating a new value for the proton mass inside the nucleus in the presence of the gravitational potential, the work has been done by using a new theory called the generalized special relativity (GSR).
\end{abstract}

Keywords: Generalized; Mass Defect; GSR; Approximations

\section{INTRODUCTION}

The simplest nucleus, that of hydrogen, is a single proton, an elementary particle of mass about $940 \mathrm{MeV}$, carrying positive charge exactly opposite to the electron's charge, having a spin of one half and being a fermion (so no two protons can be in the same quantum state).

The next simplest nucleus, called the deuteron, is a bound state of a proton and a neutron. The neutron, like the proton, is a spin one-half fermion, but it has no electric charge, and is slightly heavier (by $1.3 \mathrm{MeV}$ ) than the proton. The binding energy of the deuteron (analogous to the $13.6 \mathrm{MeV}$ for the Hydrogen atom) is $2.2 \mathrm{MeV}$. A photon of this energy could "ionize" the deuteron into a separated proton and neutron. However, it is not necessary to actually do this experiment to establish how tightly the deuteron is bound. One need only weigh the deuteron accurately. It has a mass of $1875.61 \mathrm{MeV}$. The proton has a mass of $938.27 \mathrm{MeV}$, the neutron 939.56 $\mathrm{MeV}$, so together (but some distance apart!) they have a mass of $1877.93 \mathrm{MeV}, 2.2 \mathrm{MeV}$ more than the deuteron. Thus, when a proton and a neutron come together to form a deuteron, they must unload $2.2 \mathrm{MeV}$ of energy, which they do by emitting a photon (called a $\gamma$-ray at these energies [1].
Both protons and neutrons, being fermions, obey the exclusion principle, two protons with spin up cannot be in the same state, although two with opposite spin directions could, and a proton and a neutron can occupy the same spot at the same time [2].

Protons and neutrons are referred to as nucleons. The total number of nucleons in a nucleus is usually denoted by $\mathrm{A}$, where $\mathrm{A}=\mathrm{Z}+\mathrm{N}, \mathrm{Z}$ protons and $\mathrm{N}$ neutrons. The chemical properties of an atom are determined by the number of electrons, the same as the number of protons $Z$. This is called the atomic number. Nuclei can have the same atomic number, but different numbers of neutrons. These nuclei are called isotopes, the Greek for "same place", since they are in the same place in the periodic table [3].

These nucleons attract each other with a short range but very strong force, called the nuclear force. The situation here is different from that for electrons in the atom, where the strong central force tends to dominate. In the nucleus the nucleons are attracted mainly by their immediate neighbors. Nevertheless, it is a useful beginning to think of this attractive force as being a potential well, as seen by an individual nucleon, and think in terms of the nuclei as filling the lowest available quantum states in this well, just as we did for electrons in the atom. We find, for example, that the Helium nucleus, $2 p+2 n$, is tightly bound - the four nucleons can all occupy the lowest state in the well. However, some larger nuclei, like $\mathrm{C}, \mathrm{O}, \mathrm{Fe}$ are actually a little more tightly bound even than $\mathrm{He}$ (about $8.5 \mathrm{MeV}$ per nucleon as opposed to about 7.5 for $\mathrm{He}$ ) because each nucleon is attracted to its close partners, and there are more close partners in these larger nuclei. It has also been argued that some of these higher nuclei strongly resemble bound states of $\alpha$ - particles. The total binding energy (usually expressed per nucleon) of any nucleus is easy to find-just as for the deuteron 
above, the mass of the nucleus is found accurately, and subtracted from the sum of the masses of the separate nucleons [4].

\section{GENERLIZED SPECIAL RELATIVITY (GSR) THEORY}

The Generalized Special Relativity theory is a new form of the special relativity theory that adopts the gravitational potential, and it gives the formula of relative mass to be as follows [5]:

$$
m=\frac{g_{00} m_{0}}{\sqrt{g_{00}-\frac{\mathrm{v}^{2}}{c^{2}}}}
$$

where $g_{00}=1+\frac{2 \varphi}{c^{2}}$, and $\varphi$ denotes the gravitational potential, or the field in which the mass is measured.

The derivation of the mass Eq.1 using the generalized special relativity (GSR) can be find as follows:

In the special relativity (SR), the time, length, and mass can be obtained in any moving frame by either multiplying or dividing their values in the rest frame by a factor $\gamma$.

$$
\gamma=\sqrt{1-\frac{\mathrm{v}^{2}}{c^{2}}}
$$

To see how gravity affect these quantities it is a convenient to re-express $\gamma$ in terms of the proper time [2].

$$
c^{2} d \tau^{2}=g_{\mu \nu} d x^{\mu} d x^{v}
$$

Which is a common language to both special relativity SR, and general relativity (GR). We know that in SR Eq.3 reduces to [4].

$$
\begin{gathered}
c^{2} d \tau^{2}=c^{2} d t^{2}-d x^{i} d x^{i}, x^{0}=c . t \\
\frac{d \tau}{d t}=\sqrt{1-\frac{1}{c^{2}} \cdot \frac{d x^{i}}{d t} \cdot \frac{d x^{i}}{d t}}=\sqrt{1-\frac{\mathrm{v}^{2}}{c^{2}}}=\gamma
\end{gathered}
$$

Thus we can easily generalize $\gamma$ to include the effect of gravitation by using Eq.3 and by adopting the weak field approximation where [4].

$$
\begin{gathered}
g_{11}=g_{22}=g_{33}=-1 \\
g_{00}=1+\frac{2 \varphi}{c^{2}} \\
\gamma=\frac{d \tau}{d t}=\sqrt{g_{00}-\frac{1}{c^{2}} \cdot \frac{d x^{i}}{d t} \cdot \frac{d x^{i}}{d t}}=\sqrt{g_{00}-\frac{\mathrm{v}^{2}}{c^{2}}}
\end{gathered}
$$

When the effect of motion only is considered, the ex- pression for time in SR take the form [2].

$$
d t=\frac{d t_{0}}{\sqrt{1-\frac{\mathrm{v}^{2}}{c^{2}}}}
$$

where the subscript 0 stands for the quantity measured in the rest frame. While if gravity only affect time, its expression is given by [4].

$$
d t=\frac{d t_{0}}{\sqrt{g_{00}}}
$$

In view of Eqs.8-9 and 7 the expression

$$
d t=\frac{d t_{0}}{\gamma}
$$

can be generalized to recognize the effect of motion as well as gravity on time, to get

$$
d t=\frac{d t_{0}}{\sqrt{g_{00}-\frac{\mathrm{v}^{2}}{c^{2}}}}
$$

The same result can be obtained for the volume where the effect of motion and gravity respectively reads [2].

$$
\begin{gathered}
V=V_{0} \sqrt{1-\frac{\mathrm{v}^{2}}{c^{2}}} \\
V=\sqrt{g} V_{0}=\sqrt{g_{00}} V_{0}
\end{gathered}
$$

The generalization can be done by utilizing (7) to find that

$$
V=\gamma V_{0}=\sqrt{g_{00}-\frac{\mathrm{v}^{2}}{c^{2}}} \cdot V_{0}
$$

To generalize the concept of mass to include the effect of gravitation we use the expression for the Hamiltonian in general relativity, i.e. [6].

$$
\begin{aligned}
& H=\rho c^{2}=g_{00} T^{00}=g_{00} \rho_{0}\left(\frac{d x^{0}}{d \tau}\right)^{2} \\
& =g_{00} \frac{\rho_{0} c^{2}}{\gamma^{2}}=g_{00} \frac{m_{0} c^{2}}{V_{0} \gamma^{2}}
\end{aligned}
$$

Using Eqs.14 and 15 yields

$$
\rho c^{2}=\frac{m c^{2}}{V}=\frac{g_{00} m_{0} c^{2}}{\gamma V}
$$

Therefore

$$
m=\frac{g_{00} m_{0}}{\sqrt{g_{00}-\frac{\mathrm{v}^{2}}{c^{2}}}}
$$


Which is the expression of mass in the presence of gravitational potential and it name the (GSR) theory.

In view of Eq.1, and when we substitute the value of $g_{00}$, then the relative mass according to (GSR) is find to be

$$
m=\frac{m_{0}\left(1+\frac{2 \varphi}{c^{2}}\right)}{\sqrt{1+\frac{2 \varphi}{c^{2}}-\frac{\mathrm{v}^{2}}{c^{2}}}}
$$

When the field is weak in the sense that

$$
\frac{2 \varphi}{c^{2}} \ll 1
$$

And when the speed $\mathrm{v}$ is very low such that

$$
\frac{\mathrm{v}^{2}}{c^{2}} \ll 1
$$

Eq.8 reduces to

$$
m=\frac{m_{0}\left(1+\frac{2 \varphi}{c^{2}}\right)}{\sqrt{1+\frac{2 \varphi}{c^{2}}}} \rightarrow m=m_{0} \sqrt{\left(1+\frac{2 \varphi}{c^{2}}\right)}
$$

Using the identity $(1+x)^{n} \approx 1+n . x$ for $x<<1$ one can also gets:

$$
m=m_{0}\left(1+\frac{\varphi}{c^{2}}\right)
$$

And, when the field is so strong such that

$$
\frac{2 \varphi}{c^{2}} \gg 1 \text { and } \frac{\mathrm{v}^{2}}{c^{2}} \ll 1
$$

Then Eq.18 reduces to

$$
m=m_{0} \sqrt{\frac{2 \varphi}{c^{2}}}
$$

\section{RESULTS AND DISCUSSION}

The decrease in the nucleon mass inside the nucleus is assumed to result from the fact that part of these masses is consumed to bind nucleons together. The mechanism by which these masses are converted to binding energy is not clear. One tries here to explain the mass decrease in terms of (GSR). To do this one can use the expression of the mass in Eq.24 to get:

where $\varphi_{p}$ denotes the proton nuclear force, which can be calculated using the nuclear potential $\mathrm{V}=2.818 \times$ $10^{-14}$ joules.

$$
\begin{aligned}
\varphi_{p} & =\frac{V}{m_{p}}=\frac{2.818 \times 10^{-14}}{1.67 \times 10^{-27}}, \\
\varphi_{p} & =1.67842515 \times 10^{13} \mathrm{~m}^{2} / \mathrm{sec}^{2}
\end{aligned}
$$

where the subscript $\mathrm{p}$ stands for the proton. Substitute of $\varphi_{p}$ in Eq.24 yields:

$$
\begin{aligned}
& m_{p}=1.66968853 \times 10^{-27} \mathrm{~kg} . \\
& m_{p}=1.0056 \mathrm{amu}_{\cdot}
\end{aligned}
$$

This nucleon (proton) mass value is smaller than the value of the free proton

$$
m_{p}=1.0078 \mathrm{amu} .
$$

Also the binding energy (BE) per nucleon can be calculated using the new value of the nucleon mass as follow:

$$
\Delta m_{p}=m_{p 1}-m_{p 2}=1.0078-1.005=0.0022 a m u
$$

where $m_{p 1}$ stands for the proton mass, and $m_{p 2}$ for the proton mass value using the (GSR).

$$
\begin{aligned}
B E & =\Delta E=\Delta m_{p} c^{2}=\Delta m \times 931.5 \mathrm{Mev} \\
& =0.0022 \times 931.5=2.0493 \mathrm{Mev}
\end{aligned}
$$

The Deuterium consists of one proton and one neutron. The mass of the Deuterium is:

$$
M_{D}=M_{D}\left({ }^{2} H_{1}\right)=2.0141 u
$$

Since the mass of the free neutron is:

$$
m_{n}=1.0087 \mathrm{amu}
$$

Thus the mass of the proton inside the ${ }^{2} H_{1}$ nucleus is:

$$
m_{p}=M_{D}-m_{n}=1.0054
$$

The proton mass defer it thus given by:

$$
\begin{aligned}
\Delta m_{p} & =1.0078-1.0054 \\
& =0.0024 \mathrm{amu}
\end{aligned}
$$

\section{CONCLUSIONS}

The proton mass value found by Eq.27 using the (GSR) theory, is approximately the same to that calculated in Eq.33 as a difference between the deuteron mass $\mathrm{M}_{\mathrm{D}}\left({ }^{2} H_{1}\right)$ and the neutron mass $m_{p}$. The error percentage is found to be not more than $0.2 \%$, which shows the accuracy in the calculations of the (GSR) theory. Adopting of the gravitational potential in this work presents two important results, these are, a new value for the proton (nucleon) mass inside the nucleus and the decrease in the proton mass value explains the increase in the calculated 
nucleus mass value (the mass defect).

\section{REFERENCES}

[1] Magill, J. and Galy, J. (2005) Radioactivity and radionuclide's. Institute for Trans-Uranium Elements, 32(5), 254-260.

[2] Lawden, F. and Derek (1982) An introduction to tensor calculus and relativity. John Wiley and Sons, New York.

[3] Martin, R. (2006) Nuclear and particle physics. Univer- sity College London, London.

[4] Weinberg, S. (1972) Gravitation and cosmology. John Wiley and sons, New York.

[5] Mubarak, D.A. and Ali, T. (2003) The special relativity in the presence of Gravitation and Other Field. U of K, Khartoum.

[6] Adler, R., Basin, M. and Shifter, M. (1975) Introduction to general relativity. MC Graw Hill Book Company, Tokyo. 